ples (2), verification (3), and finding an answer (4). No answers are given. There are no diagrams. A bibliography and an index of symbols are included. The typography is handsome, and the use of boldface and italic type helpful.

The authors are to be congratulated on an ambitious but successful undertaking. Their book is certain to be recognized as a valuable contribution to mathematical literature as both text and reference.

John M. H. Olmsted

Grundlagen und Anwendungen der Informationstheorie. By W. MeyerEppler. Kommunikation und Kybernetik in Einzeldarstellungen, vol. 1. Berlin-Göttingen-Heidelberg, Springer, 1959. $18+446$ pp. DM 98.

Information theory has provided mathematics with a number of interesting problems and with at least one new idea (the KolmogorovSinai invariant in ergodic theory). These matters will doubtless be of interest for some time to come; they may even find a permanent place on the mathematical scene. The volume under review, the first in Springer's new series on information and control, edited by $\mathrm{Mr}$. Meyer-Eppler, covers most of the topics usually associated with the phrase information theory in its broadest meaning. The first six chapters treat Fourier analysis of signals, channels, communication in the presence of noise, and coding theory. The last four chapters deal with the sense organs as links in a communication channel, with optics and acoustics, and with structural linguistics. Since the mathematical treatment is casual, the reader hoping to come to grips with the analytic and probabilistic problems involved will be disappointed. On the other hand, he will find a clear account of the pre-mathematical ideas, together with a vast amount of illustrative material and a large number of interesting applications of information-theoretic notions to such subjects as phonetics and phonemic analysis, morphology, semantics, and the theory of vision. For those of us who are used to reading German in just one field, the book is hard to read because of the breadth of the subject-matter; a translation would find a wide audience.

\title{
Patrick Billingsley
}

An introduction to the geometry of numbers. By J. W. S. Cassels. Grundlehren der mathematischen Wissenschaften, vol. 99. BerlinGöttingen-Heidelberg, Springer, 1959. $7+344$ pp. DM 64.50; bound, DM 69.

The geometry of numbers deals essentially with an arithmetical 
question - to find the minimum value or lower bound of a real function $f(x)$ of $n$ variables $(x)$ for integer values of the variables subject sometimes to supplementary conditions, e.g. that the variables are not all zero. The first results of any generality were due to Hermite who in 1845 began his well-known letters to Jacobi in which he expounded his new ideas and in particular, the now classic method of the variation of parameters. No other general method was discovered until Minkowski showed that many new results could be found when $f(x)$ is a homogeneous function of the variables, and the region $R$ of volume $V$ defined by $f(x) \leqq 1$ was convex and was symmetrical around the origin $O$. Define a lattice $\Lambda$ as the $n$-dimensional set of points given by

$$
x_{r}=\sum_{s=1}^{n} a_{r s} \xi_{s}, \quad(r=1,2, \cdots, n),
$$

where the coefficients $a_{r s}$ are all real and the determinant $\left\|a_{r s}\right\|=\Delta>0$, and the $\xi$ runs through all integer values. He proved the fundamental

Theorem. If $V \geqq 2^{n} \Delta$, a point of $\Lambda$ other than $O$ is contained in $R$.

His work was evidence of the great power of the geometrical ideas which he introduced. An account of his development is given in his Geometrie der Zahlen (1910) and in his Diophantische Approximationen (1907). Most of his results refer to convex regions, but it is still an exceedingly difficult problem for an $n$-dimensional convex region to replace the constant $2^{n}$ by smaller constants and of course to find the best possible constant.

Partial results for non-convex regions $R$ were found by inscribing convex regions in $R$. In particular, he considered two special problems which will be mentioned later. However, no nontrivial general results similar to those for convex regions were known for non-convex regions.

Some years after the time of Minkowski, the subject was enriched by the new ideas and methods of great depth and power found by Blichfeldt, and applicable to some of the well known problems such as the minimum value of a definite quadratic form in $n$ variables, and of the product of $n$ homogeneous linear forms. He found greatly improved results. The emphasis, however, was on convex regions.

The first real approach to non-convex regions was made by Davenport when the region $R$ is defined by

$$
f\left(x_{1}, x_{2}, x_{3}\right)=\prod_{r=1}^{3}\left|\sum_{s=1}^{3} a_{r s} x_{8}\right| \leqq 1,
$$

where either the $a_{r s}$ are all real, or the $a_{1 s}$ are real and the $a_{2 s}, a_{3 s}$ are 
conjugate complex numbers. He found the best possible result for the minimum of $f\left(x_{1}, x_{2}, x_{3}\right)$ by considering non-convex two-dimensional regions, very complicated in the second case, but this did not lead to any similar general results for non-convex regions.

The first vital breach was made by Mordell in 1940 when the region $R$ was defined by $|f(x, y)| \leqq 1$ where $f(x, y)$ is a binary cubic form with real coefficients. By means of a linear substitution with real coefficients, $f(x, y)$ can be transformed into two canonical forms. When only one root of $f(x / y, 1)=0$ is real, this can be taken as

$$
g(x, y)=x^{3}-3 x y^{2}-y^{3} .
$$

The question of finding the minimum value of $f(x, y)$ for integers $x, y$ not both zero was thus reduced to that of finding the greatest value of $\Delta$ such that every lattice of determinant $\Delta$ had a point other than the origin $O$ in the region $|g(x, y)| \leqq 1$. This was done by using ideas capable of further extensive applications. In particular, an important part in the proof was played by the critical lattices, i.e. those whose only points in $R$ were the origin and boundary points of $R$. The way was now open for further investigations. This meant that now progress could be made in dealing with non-convex regions. Then Mahler developed a general theory. He considered sequences of lattices and showed that subject to simple and general conditions, the sequence contained a convergent subsequence. His compactness theorem was fundamental and far reaching for the newer work, completely transforming the subject. Then Davenport, C. A. Rogers and others have introduced further new concepts and have found many important and striking results.

The time had become ripe for a new book on the geometry of numbers, the more so since the next book dealing with some of the subject matter, namely, Koksma's Diophantische Approximationen was published in 1936 since when the subject has been completely transformed. Then there appeared in the 1950's Keller's Geometrie der Zahlen in the Enzyklopädie, but this was not a treatise and much work has been done since it was written. Now, Dr. Cassels, who has himself made many important contributions in this field, has come on the scene and has produced the present volume. It is an excellent book and should be in the library of all interested in number theory. It is a well written book and makes pleasant reading. He has reworked and amplified many proofs giving of ten his own version, and also a unified treatment in which particular results have been dealt with in a more general and systematic way. This applies especially to some of the older results which are seen now in a modern setting. 
The book contains an account of most of the worth while results and it is really surprising what a wealth of ideas and methods occur in these proofs. It adds considerably to the interest of the book that there are a number of self-contained chapters which can be read at once by those interested in particular topics and in which others can browse. All are sure to find proofs to their liking, whether more interested in arithmetic, geometry or analysis, and will be very grateful indeed to Dr. Cassels for having dealt with all aspects of the subject.

We give a brief indication of the contents of the book. There are several chapters dealing with the modern development of problems known to Minkowski but not solved in his time. Chapter VI deals with what is now known as the theorem of Minkowski-Hlawka. The problem is to find numbers $\Delta_{1}$ such that there exist lattices $\Lambda$ of determinant $\Delta_{1}$ which are admissible for a non-convex region $R$, i.e. $\Lambda$ has no point except the origin $O$ in $R$. A conjecture of Minkowski was proved in 1944 by Hlawka who showed that if $R$ is any bounded $n$-dimensional set with Jordan volume $V_{1}$, then if $\Delta_{1}>V_{1}$, there exists a lattice $\Lambda$ admissible for $R$ with $d(\Lambda)=\Delta_{1}$. Further let $S$ be a star body, i.e. one in which $O$ is an inner point and any radius vector from $O$ meets the boundary of $S$ in either one point or none. Then if $S$ is bounded and symmetrical about $O$, it suffices if $\Delta_{1}>V / 2 \zeta(n)$ where $\zeta(n)=\sum_{r=1}^{\infty} r^{-n}$. These results were further investigated by Siegel who treated the subject in a much wider and more general setting. Other writers followed including Mahler, Davenport, C. A. Rogers, Macbeath and Schmidt.

Chapter XI deals with inhomogeneous problems. The best known of these arises from a conjecture attributed to Minkowski. Let $L_{1}(x), L_{2}(x), \cdots, L_{n}(x)$ be $n$ linear forms in $n$ variables $(x)$ with real coefficients and determinant $\Delta>0$, and let $c_{1}, c_{2}, \cdots, c_{n}$ be $n$ real numbers. The problem is to prove that there exists an integer set $(x)$ for which

$$
\prod_{r=1}^{n}\left|L_{r}(x)+c_{r}\right| \leqq 2^{-n} \Delta .
$$

The result for $n=2$ is due to Minkowski and numerous proofs have been found for $n=2$. The result for $n=3$ was first proved by Remak and a much simpler proof was found by Davenport. Later Swinnerton-Dyer and Birch also found another simple proof. Dyson proved the result for $n=4$, and no other exact result has been proved. Less perfect estimates were first found by Tchebotareff, namely $\left(2^{-n / 2}+\epsilon\right) \Delta$, and this has been improved by Mordell, Davenport and Woods. A great deal, however, remains to be done.

The newer developments also stimulated interest in other nonhomogeneous problems and a great variety of problems has resulted. 
Some of these can be dealt with by the natural extension of techniques for homogeneous problems. In particular, the so-called transference theorems associating the inhomogeneous problem with the homogeneous one now play a prominent part in the modern theory. These were initiated by Khinchine and Mahler. Others require methods for which space cannot be found, and so this chapter is more in the nature of a report but none the less interesting and valuable.

The closest packing of an $n$-dimensional region by $n$-dimensional bodies is treated in Chapter IX. The subject has long been of interest and a simple practical application would be that of putting as many oranges as possible in a box. The problem is practically equivalent to the fundamental problem of finding the minimum value of $f(x)$, really the critical lattices of a region. This can be a very difficult problem even in some of the simplest cases. It was only in 1942 that Hajos solved completely the problem of the closest packing of $n$ dimensional cubes in a box; i.e. he found the condition that a lattice $\Lambda$ should be critical for the hyper-cube $|x|<1$. Geometrical intuition and ideas seem to be very relevant for some of the problems and occasionally the arithmetic aspect seems to have disappeared. This is noticeable in the book by Fejes Toth, Lagerungen in der Ebene auf der Kugel und in Raum, 1953. Thus the result for the packing of general convex cylinders in three dimensional space is proved by the aid of lemmas based on geometry.

Another of the older topics in which substantial progress has been made is that of successive minima dealt with in Chapter VII. Let a star body be defined by $F(x)<\lambda$, where $F(x)$ is homogeneous of the first degree and so chosen that this set may be denoted by $\lambda S$ if $S$ is the set $F(x)<1$. The $k$ th successive minima $\lambda_{k}=\lambda_{k}(F, \Lambda)$ of $F$ with respect to the lattice $\Lambda$ is defined as the lower bound of the numbers $\lambda$ such that $\lambda S$ contains $k$ linearly independent lattice points. Clearly $\lambda_{1} \leqq \lambda_{2} \leqq \cdots \leqq \lambda_{n}$. For symmetric convex regions, Minkowski proved that

$$
\lambda_{1} \lambda_{2} \cdots \lambda_{n} V \leqq 2^{n} d(\Lambda),
$$

where $V$ is the volume of $F(x) \leqq 1$. His proof of this is by no means as easy as could be desired, and simpler proofs have been given by Davenport and Weyl. The result has been extended to star bodies by C. A. Rogers, Chabauty and Mahler, and many interesting results have been found.

We now turn to some of the work new in principle. In Chapter III, an account is given of the geometrical method which led Mordell to the solution of the problem of finding the best possible result for the minimum of a binary cubic, and other problems amenable to this 
method are considered. In Chapter II, an account is given of Davenport's method for finding the minimum. He showed that ideas initiated by Hermite led to a very simple arithmetical demonstration.

We have already referred to Mahler's compactness theorem. Its importance and significance may be gauged from the fact that Chapter $\mathrm{V}$ devoted to it contains 54 pages. It has proved vital in many investigations and there is a wealth of applications in many different directions.

In Mordell's treatment of the binary cubic, the success of the method depended upon the existence of automorphs of the binary cubic. Since then automorphs of various functions, especially when infinite in number, have played a prominent part in further progress. The foundations were laid by Mahler. Then Davenport and C. A. Rogers made an application showing the existence of an infinity of integer solutions for some important minimum problems. New results were also found on isolation theorems of a character similar to those found by Markoff for binary quadratic forms. The ideas arose in Davenport's solution of the minimum of the product of three linear forms.

Another development was that of reducing an $n$-dimensional problem to an $(n-1)$-dimensional problem, an idea not unfamiliar to Hermite. Thus Mordell reduced the problem of finding the minimum of a product of three linear forms already solved by Davenport to that of finding the minimum of a binary cubic form. Results were found for the minimum of a quadratic form in $n$ variables from the corresponding form in $n-1$ variables by both Mordell and Oppenheim. In particular the inhomogeneous problem for the binary quadratic received a new lease of life from the results of Davenport, Barnes and Swinnerton-Dyer, Cassels and Miss Pitman.

There are a great many other topics of which mention might have been made but the present account will give some indication of the great advances since the time of Minkowski. Still there are a large number of apparently simple concrete questions which have not yielded to repeated efforts, e.g. Littlewood's conjecture that $\min x(\theta x-y)(\phi x-z)=0$ where $\theta, \phi$ are irrational numbers and $x, y, z$ take integer values. Simple four-dimensional problems such as the minimum of the product of four linear forms, and also the critical lattice for the region $x^{2}+y^{2} \leqq 1, z^{2}+w^{2} \leqq 1$, defy solution. A host of problems connected with the minimum of quadratic forms await solution.

Cassels' book will give a great impetus to the further study of problems associated with the geometry of numbers. One will look forward with considerable interest to future developments. 